\title{
Effects of an intra-ventricular assist device on the stroke volume of failing ventricle: Analysis of a mock circulatory system
}

\author{
Shidong Zhu ${ }^{\mathrm{a}}$, Lin $\mathrm{Luo}^{\mathrm{a}}$, Bibo Yang ${ }^{\mathrm{b}}$, Kai Ni ${ }^{\mathrm{a}}$, Qian $\mathrm{Zhou}^{\mathrm{a}}$, Xinghui $\mathrm{Li}^{\mathrm{a}, *}$ and \\ Xiaohao Wang ${ }^{\text {a,* }}$ \\ ${ }^{a}$ Division of Advanced Manufacturing, Graduate School at Shenzhen, Tsinghua University, Shenzhen \\ 518055, Guangdong, China \\ ${ }^{\mathrm{b}}$ Cardiac Surgery Center, Beijing Anzhen Hospital, Capital Medical University, Beijing 100029, China
}

\begin{abstract}
.
BACKGROUND: A novel intra-ventricular assist device (iVAD) was established as a new pulsatile assist device to address various disadvantages, such as bulky configuration and reduced arterial pulsatility, observed in conventional ventricular assist devices.

OBJECTIVE: Analyzed the native left ventricular stroke volume (SV) after iVAD support in vitro.

METHODS: The SV of iVAD was examined in a home-designed mock circulatory system (MCS) at different heart rates and drive pressures and the SV of a failure ventricle was examined with iVAD at 75, 90, $120 \mathrm{bpm}$ and 120-180 $\mathrm{mmHg}$ drive pressure after iVAD support. Data pertaining to native left ventricular SV before and after iVAD support were compared.

RESULTS: The native ventricular SV was improved by iVAD when its drive pressure (DP) was slightly greater than that of the mock system. Conversely, the native ventricular SV was decreased when DP was much greater than that (150 $\mathrm{mmHg}) \mathrm{of} \mathrm{MCS}$. A high DP had a significant effect on SV.

CONCLUSIONS: The proposed device improved the dysfunctional native left ventricular SV when DP of iVAD was slightly greater than that of MCS. However, iVAD reduced the SV when the drive pressure was greater than that of MCS.
\end{abstract}

Keywords: Left ventricular assist device, mock circulatory system, pulsatile pump, stroke volume

\section{Introduction}

Left ventricular assist devices (LVADs) are increasingly accepted for patients with failing hearts. These devices can be divided into two main categories: pulsatile flow (PF) LVAD and continuous flow (CF) LVAD. The advantage of PF LVADs is their ability to maintain physiological PF, but these devices have high rates of complications, such as infections and mechanical failure, because of their large size and attachments. Inlet and outlet valves are required to ensure their correct unidirectional flow [1]. Although CF LVADs work by either an axial or a centrifugal mechanism are smaller. However, they usually

\footnotetext{
${ }^{*}$ Corresponding author: Xinghui Li, A-216, Tsinghua Campus, The University Town, Shenzhen 518055, Guangdong, China. Tel.: +86 13615541349; Fax: +86 755 26036618; E-mail: li.xinghui@sz.tsinghua.edu.cn; Xiaohao Wang, A-216, Tsinghua Campus, The University Town, Shenzhen 518055, Guangdong, China. E-mail: xhwang@ mail.tsinghua.edu.cn.
}

0928-7329/18/\$35.00 (C) 2018 - IOS Press and the authors. All rights reserved

This article is published online with Open Access and distributed under the terms of the Creative Commons Attribution NonCommercial License (CC BY-NC 4.0). 


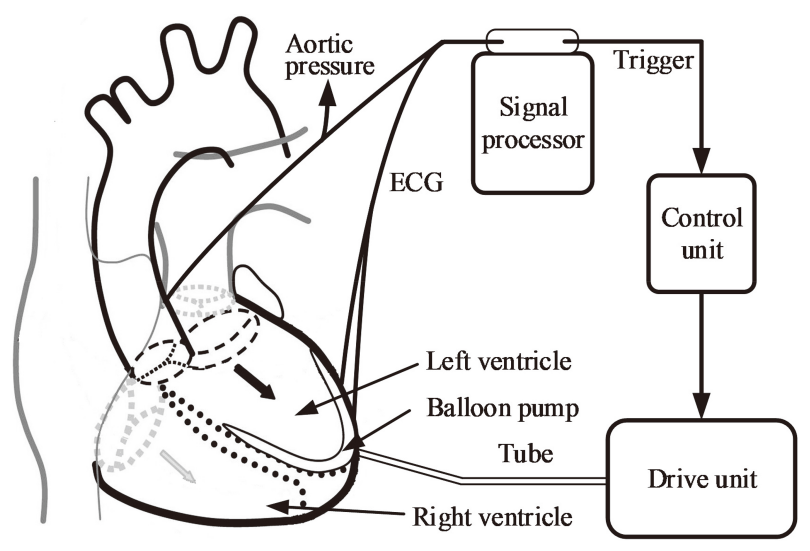

Fig. 1. Schematic of a typical intra-ventricular assist device.

provide continuous blood flow that differs from the physiological flow in terms of its recued pulsatility. The lack of pulsatility results in a sustained non-physiological pressure at the aortic root, thereby leading to aortic root dilatation and valve fusion; thus, insufficiency, platelet dysfunction, endothelial dysfunction, and gastro-intestinal bleeding occur [2-4]. Implantation with CF LVADs may contribute to the onset of right heart failure because of the rapid unloading of the left ventricle and hence causes septal shift and right ventricular dilatation. The ability of PF LVADs to unload the left ventricle is higher than that of CF LVADs [3]. Although the effect of non-physiological pulseless blood flow on the circulation and organ perfusion remains debatable, researchers suggested that PF should be preserved [5-8].

Although traditional pumps have many disadvantages, they can produce physiological flow. Several new pulsatile devices, such as C-Pulse [9,10], LibraHeart pulsatile LVAD system [11], and rotary piston blood pumps [12,13], have been designed. Therefore, pulsatile pumps should be further investigated.

In this study, a new pulsatile assist device was developed to overcome the challenges associated with traditional pulsatile pumps and was examined in a mock circulatory system (MCS) in vitro. The special intraventricular co-pulsatile mode of the proposed assist device might affect the SV of the native ventricle. This study aimed to assess the changes in the stroke volume (SV) of the left failure ventricle with the new device support.

\section{Materials and methods}

\subsection{A novel intra-ventricular assist device}

An intra-ventricular assist device (iVAD) is a new pulsatile balloon pump driven by a pneumatic drive unit or an electric drive unit and is implanted in the ventricle without the use of mechanical heart valves. Figure 1 shows the principal components of the pump and their functions. The iVAD mainly consists of a balloon pump, a signal processor, a control unit, and a drive unit. The balloon pump is implanted into the left ventricle starting from the apex. In this study, the minimum volume of iVAD is $6.3 \mathrm{~cm}^{3}$, the balloon volume is $40 \mathrm{~cm}^{3}$, the diameter is $1.2 \mathrm{~cm}$, and the length is $5.6 \mathrm{~cm}$. During the systole, the balloon is inflating, and pushes the blood in the left ventricle to increase the left ventricular pressure. When during the diastole, the balloon is deflating which is benefit to the blood flow into the left ventricular cavity. The balloon pump contains a multi-layer medical polyurethane (PU) and silicone plastic polymer 


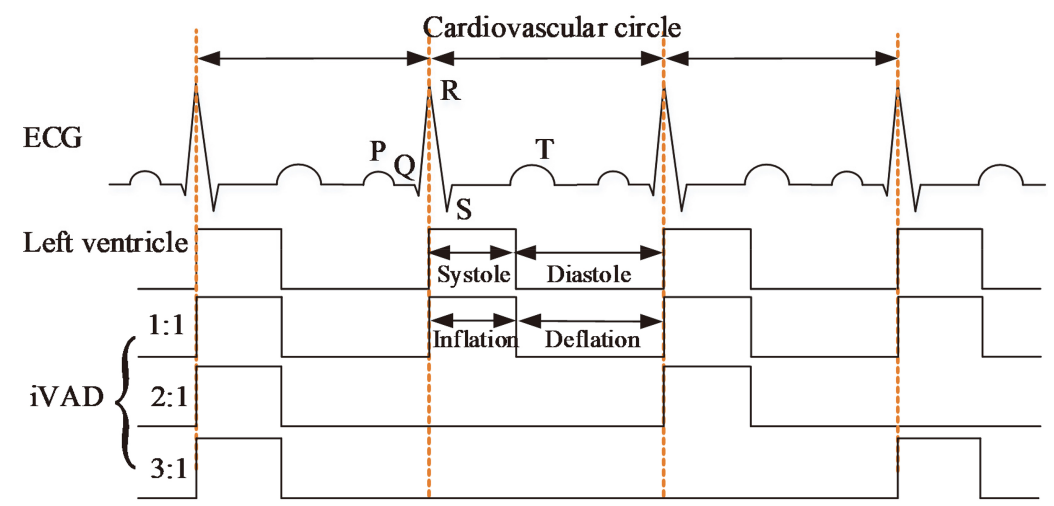

Fig. 2. Illustration of the pulsatile rhythm of the left ventricle and the intra-ventricular assist device.

materials and resembles an ellipsoid with a mouth opening at the bottom. This part is implanted into the left ventricle starting from the apex. The iVAD assists the ventricle of patients with HF to pump blood. The innermost layer of the balloon is in direct contact with gas, such as air or He. Nevertheless, the outermost layer covered with biological materials is in contact with blood, and composed of medical polyurethane (PU) from Bayer (Leverkusen, Germany) and characterized by optimum blood and tissue compatibility. The iVAD yields a smaller implanted volume and creates a smaller operation wound than that of traditional PF LVAD. In contrast to the continuous workload of CF-LVADs, iVAD can work intermittently.

The working approach of an iVAD is relatively similar to that of an IABP [14,15] and a C-Pulse [16]. Inflated and deflated phases are in their opposite direction. The co-pulsation of iVAD synchronizes with an electrocardiogram (ECG) and a dicrotic notch or phonocardiographs. ECG signals are collected directly from two electrodes which are placed on the surface of the myocardium. The micro-ECG is amplified and filtered by a signal processor. The $\mathrm{R}$ wave peaks detected from ECG by a peak detection algorithm are used to trigger pulses, which drives pump inflation via a control unit. Whereas the dicrotic notch peaks collected from the aortic arch trigger iVAD deflation. In this study, the inflation and deflation of the balloon pump were controlled by the electropneumatic regulators (ITV2030-312BL, SMC Pneumatics, Tokyo, Japan) and the vacuum proportional valves (ITV2090-31N2BN5, SMC Pneumatics, Tokyo, Japan), respectively. A selector valve (MHE4-MS1H-3/2G-QS-8-K, Festo, Esslingen, Germany) was employed to switch to inflated phase or deflated phase. The positive and negative drive pressure of the iVAD are adjustable by the voltage of proportional valve in the range of $40-500 \mathrm{mmHg}$ and vacuum pressure $20-100 \mathrm{mmHg}$. In some patients, the incisure points are not well defined, therefore the phonocardiograph is used to trigger deflation that considers the second heart sound as the time of the aortic valve closure. Figure 2 presents the pulsatile time relation between the left ventricle and iVAD. The drive modes mainly include 1:1, 2:1, and 3:1 (ventricular beats: iVAD beats). The assist mode is adjusted according to the heart rate (HR). For example, the assist mode is set to $3: 1$ when HR is 120 beats per minute (bpm) under a sufficient blood supply. In this study, ECG was provided by a generator (SKX-2000SUP+, Mingsheng, China). To facilitate the experiment, we defined the systolic period as 0.35 times of the cardiac cycle.

\section{2. $M C S$}

MCS comprises systemic and pulmonary circulation is designed to examine the performance of iVAD. Figure 3 shows the schematic of MCS. Four flexible PU sacs were employed as left ventricle, right ventri- 


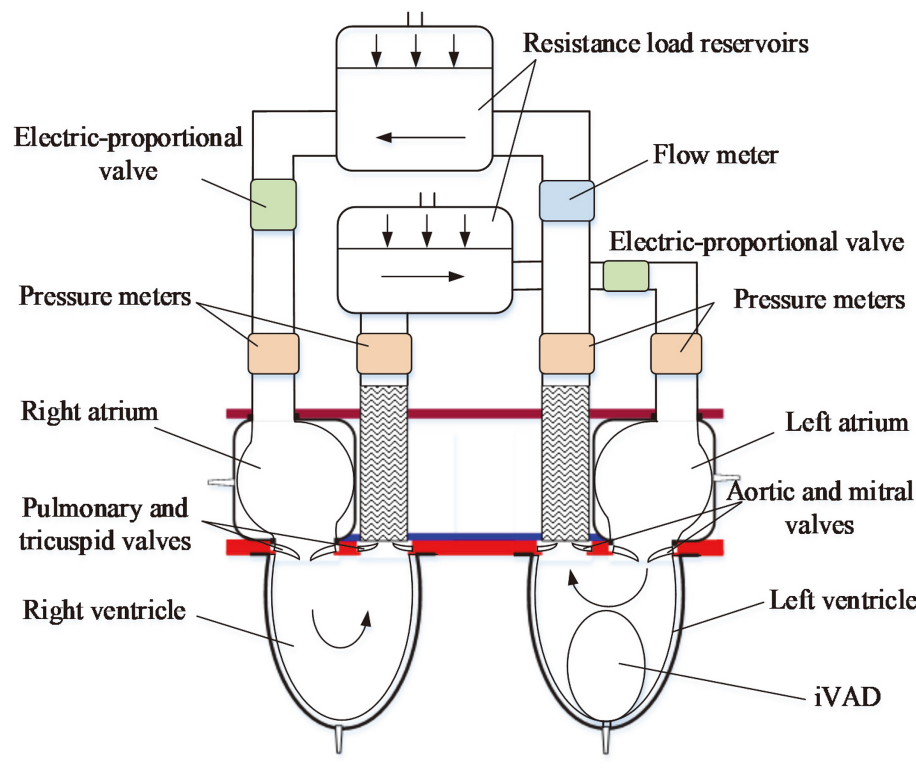

(a)

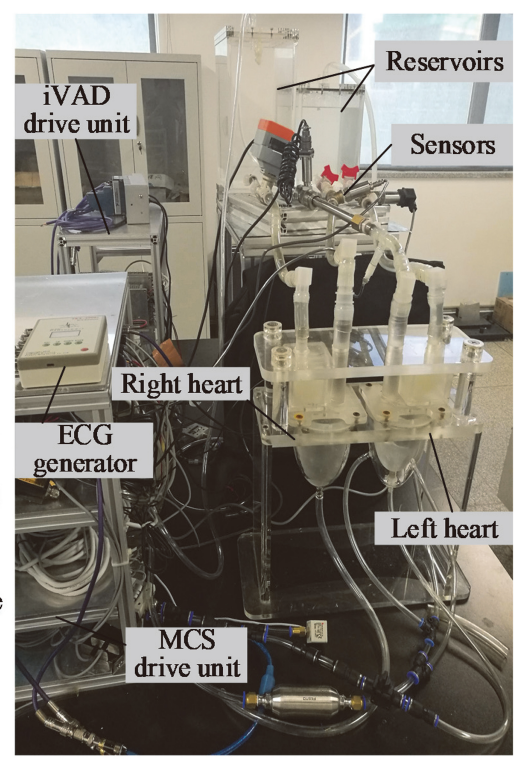

(b)

Fig. 3. (a) Schematic of an experimental mock cardiovascular system, (b) Picture of the experimental setup.

cle, left atrium, and right atrium. In the MCS, the volume of ventricular sacs is $180 \mathrm{~cm}^{3}$. These sacs were housed in pressurization chambers composed of transparent acrylic. Ventricular systole was controlled with two electropneumatic regulators and 3/2 way solenoid valves (VT325-025GS, SMC Pneumatics, Tokyo, Japan) to provide passively filled heart chambers and contractility. The term ventricular compliance refers to the distensibility of the relaxed ventricle, in a general sense, defined in terms of its diastolic pressure-volume relationship. During ventricular diastole, the ventricle sacs is deflating by controlling of two vacuum proportional valves The systolic period was determined by the duty cycle of the selector valve, which was triggered by the ECG R peaks from the generator. Because of the small resistances of the native valves (about $0.004 \mathrm{mmHg} / \mathrm{ml}$ ), the general one-way valve cannot present the native valves due to the relatively high resistance. Therefore, the artificial mechanical valves were used as aortic, pulmonary (SJM 23AJ-501, St. Jude Medical, USA), mitral, and tricuspid valves (SJM 27MJ-501, St. Jude Medical, USA). Contraction and pulsatile flow were sustained by connecting the chambers between acrylic cavities and sacs to the pneumatic controller. The resistance load reservoirs were employed to imitate vascular resistance and compliance. The compressible gas above the fluid in the reservoirs controlled by electropneumatic regulators represent the peripheral resistance (afterload), and represent the circulation compliance. The normal afterload was set to $80 \mathrm{mmHg}$, for the HF patients, the afterload value is increased. From the Windkessel et al. [17] elastance model and Bowles et al. [18] theoretical analysis model, the relationship between the initial volume of the sealed gas and the physiological value and the mean aortic pressure as following.

$$
V_{0}=n \cdot C_{a o} \cdot\left(P_{a o, m}+P_{a t m}\right)
$$

Where $V_{0}$ is the initial volume of the sealed gas, $n$ is the gas constant (1.1), $P_{a o, m}$ is the mean aortic pressure, and $P_{\text {atm }}$ is the atmospheric pressure $(760 \mathrm{mmHg})$. Normal aortic compliance is about $2 \mathrm{ml} / \mathrm{mmHg}$, for patients with HF, the compliance is $1.8 \mathrm{ml} / \mathrm{mmHg}$ which is less than that of normal.

The electric-proportional valve was placed next to the resistance chambers to adjust the preload in pulmonary and systemic circulation components. All of the connections between chambers and reservoirs 
were fabricated with tygon tubing. The flow rate was examined with FD-SS20A (KENYENCE, Japan), whereas pressure was determined by high-frequency dynamic response micro-electro-mechanical system sensors CYY8 (Xian HangDong, China). The whole system was filled with water, which mimicked blood. The control and acquisition of the flowmeter and pressure meter data signals were achieved at $1 \mathrm{kHz}$ with a custom system (National Instruments, Austin, TX, USA). All of the signals were filtered through a second-order Butterworth low-pass filters with $20 \mathrm{~Hz}$ cutoff frequency.

\subsection{Testing}

The balloon pump was implanted in the MCS (Fig. 3) without the left ventricular membrane to examine the input and output dynamics of iVAD. The circulatory loop parameters were as follows: the preload and afterload of systemic circulation in the MCS of 15 and $80 \mathrm{mmHg}$, respectively; HR of $60 \mathrm{bpm}$; and drive pressure of $120 \mathrm{mmHg}$. The total volume of the experimental balloon of iVAD was $40 \mathrm{~cm}^{3}$. In other experiments, HR varied from $60 \mathrm{bpm}$ to $130 \mathrm{bpm}$ at $5 \mathrm{bpm}$ intervals, and the drive pressures were $120,150,180$, and $200 \mathrm{mmHg}$, respectively. Duration of systolic phase (iVAD inflation) was defined as the $32 \%$ of cardiac cycle, and the remaining $68 \%$ was defined as the diastolic phase (iVAD deflation).

MCS was used to simulate a healthy heart status. HR was set to $75 \mathrm{bpm}$, the drive pressure of MCS $\left(P_{\text {mock }}\right)$ was fixed at $140 \mathrm{mmHg}$, and the afterload was adjusted to $80 \mathrm{mmHg}$. In addition, in HF condition, the native ventricle weakened and was unable to contract adequately to pump sufficient blood amount, Cardiac output (CO) and arterial pressure decreased to below normal, HR is higher than that of healthy level at rest, and the left ventricular systolic pressure (LVSP) is less than normal value. In this experiment, HR was set to 75, 90, and $90 \mathrm{bpm}$, and $P_{\text {mock }}$ was set to $110 \mathrm{mmHg}$. The systolic period is $32 \%$ of cardiac cycle. Furthermore, The MCS was used to evaluate the balloon pump of iVAD implanted in the left ventricular sac starting from the apex under HF conditions. The drive pressures of iVAD $\left(P_{i V A D}\right)$ were set to 120,150 , and $180 \mathrm{mmHg}$ at 75,90 , and $120 \mathrm{bpm} \mathrm{HR}$, respectively. The iVAD systole was the same as above.

\section{Results and discussion}

The mean SV of iVAD under different conditions is shown in Fig. 4. The mean SV was determined as an average of 20 cardiac cycles in each case. In Fig. 4, SV decreased as HR increased but increased as the of the drive pressure increased (the legend in Fig. 4 represents the drive pressure). SV reached the maximum when $\mathrm{HR}$ is $60 \mathrm{bpm}$, such as $53 \mathrm{ml}$ and $45 \mathrm{ml}$ at 180 and $200 \mathrm{mmHg}$ drive pressure. SV is greater than the volume of the balloon pump $\left(40 \mathrm{~cm}^{3}\right)$ due to the plastic deformation of the balloon. Which will cause the balloon to fatigue and reduce its life.

Figure 5 illustrates the hemodynamics of heart failure and iVAD support in MCS including ECG, left ventricular pressure (LVP), aortic pressure (AoP), iVAD drive pressure synchronous drive pulse, aortic flow $(\mathrm{AoF})$, and MCS drive pressure $\left(P_{\text {mock }}\right)$. As shown in the figure, the morphology characteristics of the waveforms are similar to those of the native physiological curve. The left ventricular pressure curve likes plateau form. However, at the the end-systole and diastole, there are some differences between AoP curve and physiological curve. AoP, followed by decreasing ventricular pressure, substantially dropped in amplitude at mechanical aortic valve closure during terminal systole because of aortic valve insufficiency and lack of systemic circulatory compliance. The typical waveforms (HR $90 \mathrm{bpm}, P_{i V A D} 150 \mathrm{mmHg}$ ) of iVAD support are shown in Fig. 5. LVP, AoP, and SV were improved 


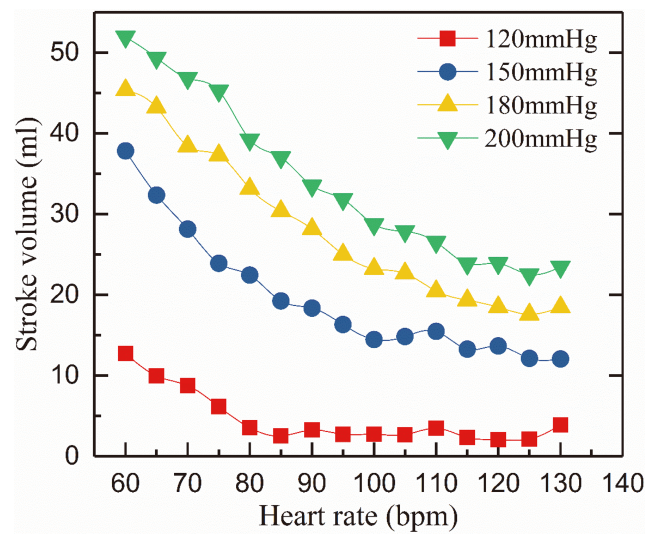

Fig. 4. Relation of iVAD stroke volume and heart rate at a drive pressure of 120-200 $\mathrm{mmHg}$ and an afterload of $80 \mathrm{mmHg}$.
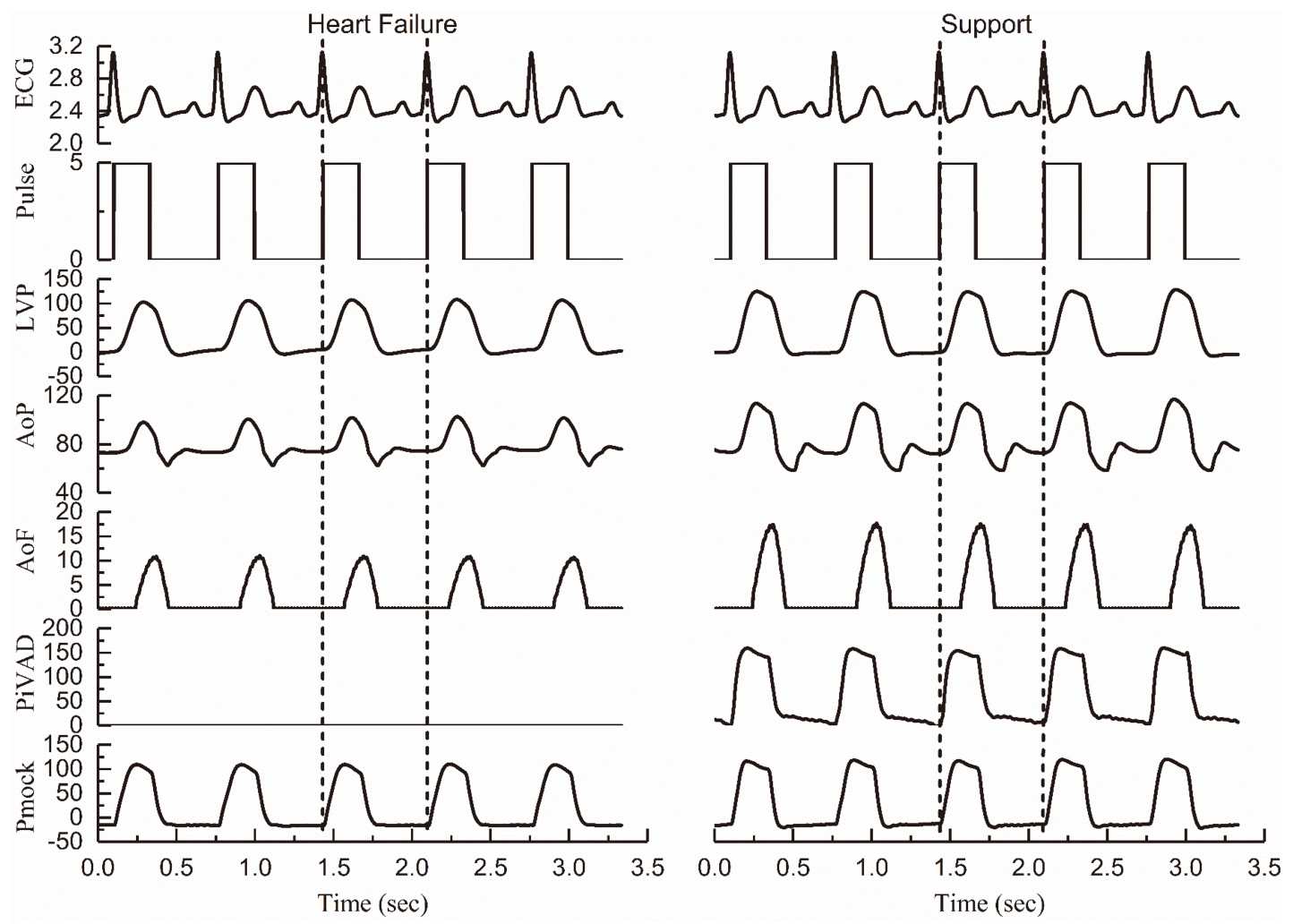

Fig. 5. Comparison of the hemodynamics of failure heart and an iVAD support (ECG: V; Pulse: V; LVP: mmHg; AoP: mmHg; AoF: L/min; PiVAD: mmHg; Pmock: $\mathrm{mmHg}$ ).

compared with those of a failure heart. Table 1 describes in detail the value of hemodynamics under different conditions. In healthy condition, LVSP, the systolic aortic pressure (SAP), and the diastolic aortic pressure (DAP) are 128, 121, $84 \mathrm{mmHg}$. SV is $41.6 \mathrm{ml}$. These findings are consistent with the healthy hemodynamics, and SV is slightly lower than that of a healthy heart at rest (50-70 ml). In HF conditions, a low drive pressure $(110 \mathrm{mmHg})$ reduces the ventricular systolic capacity to simulate heart failure at 
Table 1

Hemodynamics of failure heart with iVAD in a mock cardiovascular system

\begin{tabular}{|c|c|c|c|c|c|c|c|c|c|c|c|c|c|}
\hline Parameter & $\mathrm{H}$ & & $\mathrm{HF}$ & & & & & & uppor & & & & \\
\hline HR (bpm) & 75 & 75 & 90 & 120 & & 75 & & & 90 & & & 120 & \\
\hline$P_{i V A D}(\mathrm{mmHg})$ & - & - & - & - & 120 & 150 & 180 & 120 & 150 & 180 & 120 & 150 & 180 \\
\hline LVSP (mmHg) & 128 & 107 & 106 & 106 & 118 & 139 & 155 & 117 & 142 & 158 & 116 & 140 & 156 \\
\hline $\mathrm{SAP}(\mathrm{mmHg})$ & 121 & 103 & 102 & 100 & 114 & 139 & 143 & 112 & 133 & 146 & 111 & 129 & 145 \\
\hline DAP (mmHg) & 84 & 74 & 77 & 74 & 77 & 84 & 87 & 76 & 89 & 92 & 81 & 90 & 93 \\
\hline$S V_{i V A D}(\mathrm{ml})$ & - & - & - & - & 6.18 & 23.9 & 37.3 & 3.27 & 18.4 & 28.2 & 2.09 & 13.7 & 22.5 \\
\hline$S V_{\text {theo }}(\mathrm{ml})$ & - & - & - & - & 35.1 & 49.4 & 56.1 & 32.2 & 43.9 & 47 & 30.9 & 39.2 & 41.3 \\
\hline$S V_{H F} / \mathrm{SV}(\mathrm{ml})$ & 41.6 & 28.9 & 25.5 & 18.8 & 38.6 & 46.2 & 54.4 & 31.9 & 37.1 & 44 & 23.3 & 27.3 & 34.2 \\
\hline SV (ml) & - & - & - & - & 32.4 & 22.3 & 17.1 & 28.6 & 18.7 & 15.8 & 21.2 & 13.6 & 11.7 \\
\hline $\mathrm{g}(\%)$ & - & - & - & - & 12.2 & 22.8 & 40.8 & 12.3 & 26.7 & 38.0 & 12.8 & 27.7 & 37.8 \\
\hline Rise or fall & - & - & - & - & $\uparrow$ & $\downarrow$ & $\downarrow$ & $\uparrow$ & $\downarrow$ & $\downarrow$ & $\uparrow$ & $\downarrow$ & $\downarrow$ \\
\hline
\end{tabular}

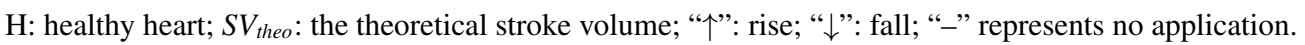

75, 90 and $120 \mathrm{bpm}$. The left ventricular pressure (LVP) decreases, LVSP reduces from $128 \mathrm{mmHg}$ to $107 \mathrm{mmHg}$, and the corresponding aortic blood pressure (AoP) decreases at $75 \mathrm{bpm}$. With the increase in HR, LVAP, SAP, and DAP are essentially unchanged, but SV decreases, such as $18.8 \mathrm{ml}$ at $120 \mathrm{bpm}$

In iVAD support conditions, iVAD improved LVSP, SAP, DAP, and SV. In Table $1, S V_{\text {theo }}$ is the theoretical stroke volume of a failing ventricle with iVAD support, and $S V$ represents the change in stroke volume.

$$
\begin{aligned}
S V_{\text {theo }} & =S V_{i V A D}+S V_{H F} \\
\Delta S V & =S V-S V_{i V A D}
\end{aligned}
$$

Where $S V_{i V A D}$ is the stroke volume of the iVAD, $S V_{H F}$ is the stroke volume of a failing ventricle at 75 , 90 , and $120 \mathrm{bpm}$, and $S V$ is the total stroke volume of a failing heart with iVAD support. g represents the rise or fall percentage of stroke volume at different HR and drive pressure.

$$
\varepsilon=\left(\Delta S V-S V_{H F}\right) / S V_{H F} \times 100 \%
$$

Where the positive and negative symbols of Eq. (4) represent an increase or a decrease in value. "个" represents increase, and " $\downarrow$ ” represents decrease. At a drive pressure of $120 \mathrm{mmHg}$, iVAD improved the SV of the native ventricle, and the percentage increased slightly as HR increased. However, the SV of the native left ventricle after iVAD support was less than that of $S V_{\text {theo }}$, the greater the percentage of reduction with $P_{i V A D}$ increasing, and at the some drive pressure, the greater HR, the greater the percentage drop. At a drive pressure of $180 \mathrm{mmHg}$ and HRs of 75 and $90 \mathrm{bpm}$, the sac employed as the left ventricle produced an expanded plastic deformation caused by iVAD drive pressure. As drive pressure increased, the percentage increased, and the maximum of which reached $40.8 \%$. The MCS experimentally revealed that iVAD affected the SV of the native dysfunctional ventricle. The iVAD was conducive to left ventricular stroke when the drive pressure of iVAD was slightly greater than that of MCS $(120 \mathrm{mmHg})$. However, the drive pressure of iVAD was much greater than that of MCS, and iVAD support attenuated the SV of the native ventricle. For example (Fig. 5), LVSP reached $142 \mathrm{mmHg}$ and the total SV was $37.1 \mathrm{ml}$ when the drive pressure of iVAD was $150 \mathrm{mmHg}$. The native left SV $(18.7 \mathrm{ml})$ was less than $25.5 \mathrm{ml}$. Although iVAD improved LVSP, SAP, and SV to obtain a healthy level, this device decreased the native left ventricular SV in the MCS because of a co-pulsatile in the ventricle.

In MCS, the left ventricular sac was deflating controlled by a vacuum proportion valve. The vacuum pressure was set to $30 \mathrm{mmHg}$ at different conditions and the preload was constant. Therefore, we assume that the left ventricular volume is the same at the end-diastole. $S V$ is greater than $S V_{\text {theo }} 120 \mathrm{mmHg}$ drive 
pressure in Table 1, which revealed that the left ventricular volume under iVAD support is less than that of HF condition at the end-systole. However, in other cases, the results are just the opposite. LVP and is increased with iVAD drive pressure increasing. The increased stress on the left ventricular cavity surface inhibited the ventricular contraction. As the drive pressure difference of MCS and iVAD increases, the inhibitory effect on ventricular contraction is strong. Although, the parameters such as LVP and the ventricular volume is in the normal physiological range under iVAD support, the support mode may be detrimental to the myocardium. The specific effect of iVAD on ventricular SV requires further animal experimental studies because that differences between MCS and cardiovascular system remain uncertain.

\section{Conclusions}

An iVAD implanted in the left ventricle can improve LVSP, SAP, and SV. With this support, hemodynamics reached a healthy level in the MCS. The proposed device improved the dysfunctional native left ventricular SV when the drive pressure of iVAD was slightly greater than that of MCS. However, iVAD reduced the SV of the native ventricle when the drive pressure was greater than the pressure of MCS. A high drive pressure of iVAD corresponds to a remarkable decrease in SV of the native dysfunctional heart.

\section{Conflict of interest}

None to report.

\section{References}

[1] Ferrari M, Kruzliak P, Spiliopoulos K. An insight into short- and long-term mechanical circulatory support systems. Clin Res Cardiol. 2015; 104: 95-111.

[2] Hiraoka A, Cohen JE, Shudo Y, MacArthur JW, Howard JL, Fairman AS, et al. Evaluation of late aortic insufficiency with continuous flow left ventricular assist device. Eur J Cardio-Thorac. 2015; 48: 400-6.

[3] Schumer EM, Ising MS, Slaughter MS. The current state of left ventricular assist devices: challenges facing further development. Expert Rev Cardiovasc Ther. 2015; 13: 1185-93.

[4] Wong K, Samaroo G, Ling I, Dembitsky W, Adamson R, del Alamo JC, et al. Intraventricular flow patterns and stasis in the LVAD-assisted heart. J Biomech. 2014; 47: 1485-94.

[5] Bozkurt S, van Tuijl S, Schampaert S, van de Vosse FN, Rutten MC. Arterial pulsatility improvement in a feedbackcontrolled continuous flow left ventricular assist device: an ex-vivo experimental study. Med Eng Phys. 2014; 36: 128895.

[6] O'Neil MP, Fleming JC, Badhwar A, Guo LRR. Pulsatile Versus Nonpulsatile Flow During Cardiopulmonary Bypass: Microcirculatory and Systemic Effects. Ann Thorac Surg. 2012; 94: 2046-53.

[7] Wang S, Izer JM, Clark JB, Patel S, Pauliks L, Kunselman AR, et al. In Vivo Hemodynamic Performance Evaluation of Novel Electrocardiogram-Synchronized Pulsatile and Nonpulsatile Extracorporeal Life Support Systems in an Adult Swine Model. Artif Organs. 2015; 39: E90-E101.

[8] Alkan T, Akcevin A, Undar A, Turkoglu H, Paker T, Aytac A. Effects of pulsatile and nonpulsatile perfusion on vital organ recovery in pediatric heart surgery: a pilot clinical study. Asaio J. 2006; 52: 530-5.

[9] Rame JE, Georgakopoulos D, Pomfret D, Atluri P, Wiegn P, Segers P, et al. Arterial and Cardiac Hemodynamics in Advanced HF Patients Implanted With the C-Pulse Counterpulsation Device: Implications for Myocardial Recovery. Circulation. 2015; 132.

[10] Zeriouh M, Sabashnikov A, Bowles CT, Weymann A, Ghodsizad A, Mohite PN, et al. Full-Support LVAD Implantation in a C-Pulse Heart Assist System Recipient with Deteriorating Chronic Heart Failure: Is It Feasible and Safe? Asaio J. 2016; 62: E55-E7. 
[11] Choi SW, Nam KW, Lim KM, Shim EB, Yong SW, Woo HM, et al. Effect of counter-pulsation control of a pulsatile left ventricular assist device on working load variations of the native heart. Biomedical Engineering Online. 2014; 13: 1-11.

[12] Wappenschmidt J, Autschbach R, Steinseifer U, Schmitz-Rode T, Margreiter R, Klima G, et al. Rotary piston blood pumps: past developments and future potential of a unique pump type. Expert Rev Med Devic. 2016; 13: 759-71.

[13] Wappenschmidt J, Sonntag SJ, Buesen M, Gross-Hardt S, Kaufmann T, Schmitz-Rode T, et al. Fluid Dynamics in Rotary Piston Blood Pumps. Ann Biomed Eng. 2017; 45: 554-66.

[14] Ferrari G, Khir AW, Fresiello L, Di Molfetta A, Kozarski M. Hybrid Model Analysis of Intra-Aortic Balloon Pump Performance as a Function of Ventricular and Circulatory Parameters. Artif Organs. 2011; 35: 902-11.

[15] Khir AW, Bruti G. Intra-aortic balloon shape change: effects on volume displacement during inflation and deflation. Artif Organs. 2013; 37: E88-95.

[16] Slaughter MS, Cecere R, Sun B, Huprikar S, Hotz H. C-Pulse (R) System Extra-Aortic Counterpulsation for Heart Failure: Driveline Infections and Management. J Heart Lung Transpl. 2015; 34: S224-S.

[17] Westerhof N, Lankhaar JW, Westerhof BE. The arterial Windkessel. Med Biol Eng Comput. 2009; 47: 131-41.

[18] Bowles CT, Nishimura K, Shah SS, Cumming DVE, Pepper JR, Yacoub MH, et al. Development of Mock Circulation Models for the Assessment of Counterpulsation Systems. Cardiovasc Res. 1991; 25: 901-8. 